\title{
SENTIMENTOS DAS MÃES DE CRIANÇAS COM PARALISIA CEREBRAL À LUZ DA TEORIA DE MISHEL ${ }^{a}$
}

The mothers of children with cerebral palsy feelings motivated by the mishel's theory

Sentimientos de las madres de niños con parálisis cerebral a luz de la teoría de mishel

Kamilla de Mendonça Gondim

Zuila Maria de Figueiredo Carvalho

\section{RESUMO}

As crianças com paralisia cerebral sofrem com as consequências da lesão ao cérebro, apresentando dificuldades que afetam as atividades da vida diária. A família, principalmente a mãe, deve receber apoio dos profissionais para enfrentar o problema. Este estudo objetivou analisar a incerteza das mães quanto à doença de seus filhos com base na Teoria da Incerteza na Doença de Mishel. É um estudo descritivo e qualitativo, realizado em duas instituições públicas especializadas em distúrbios neuromotor da cidade Fortaleza, nos meses de julho e agosto de 2009. Foram entrevistadas 12 mães de crianças com paralisia cerebral por meio de um formulário com as variáveis sócio-econômico-sanitário-demográficas e um roteiro de entrevista semiestruturado baseado na Teoria de Mishel, na versão pais/filhos. Constatamos que as mães apresentam muitas incertezas, principalmente quanto ao futuro da criança. Tais incertezas podem ser reduzidas mediante apoio e orientação dos profissionais de saúde, melhorando, assim, a qualidade de vida de todos os envolvidos.

Palavras-chave: Paralisia cerebral. Comportamento materno. Teoria de enfermagem.

\begin{abstract}
The children with cerebral palsy suffer with the consequences of the injury to the brain, presenting difficulties that affect the activities of a daily life. The family, especially the mother, should receive support from the professionals to confront the problem. The objective is to analyze the mothers' uncertainty facing their children disease, based on the fundamental principle of Mishel's Theory of Uncertainty in Illness. It is a descriptive and qualitative study, carried out in two public institutions specialized in neuromotor disorders in Fortaleza, between July and August of 2009. Twelve mothers of children with cerebral palsy were interviewed through the use of a form with social, economic, sanitary and demographic information and a semi-structured interview based on Mishel's theory Mishel, on a parent / child version. We have noticed that the mothers show uncertainty feelings, especially regarding the child's future. Such uncertainties can be reduced by the professionals of health support and orientation, thus improving the life quality of all involved.
\end{abstract}

Keywords: Cerebral palsy. Maternal behavioral. Nursing theory.

\section{Resumen}

Los niños con parálisis cerebral sufren con las consecuencias de la lesión al cerebro, presentando dificultades que afectan las actividades de vida diaria. La familia, principalmente la madre, debe recibir apoyo de los profesionales para enfrentar el problema. Se objetivó analizar la incerteza de madres cuánto a la enfermedad de sus hijos basada en la Teoría de la Incertidumbre frente a la Enfermedad de Mishel. Se trata de un estudio descriptivo y cualitativo realizado en dos instituciones públicas especializadas en disturbios neuromotores en la ciudad de Fortaleza, en los meses de Julio y Agosto de 2009. Fueron entrevistadas 12 madres de niños con parálisis cerebral a través de un formulario con las variables socio-económico-sanitariodemográficas y un rutero de entrevista seme estructurada basada en la Teoría, versión padres/hijos. Constatamos que las madres presentan muchas incertidumbres, principalmente cuánto al futuro del niño. Tales incertidumbres pueden ser reducidas mediante apoyo y orientación de los profesionales de salud, mejorando, así, la calidad de vida de todos los envueltos.

Palabras clave: Parálisis cerebral. Comportamiento materno. Teorías de enfermería.

${ }^{1}$ Enfermeira. Mestre em Enfermagem pela UFC. Professora substituta do DENF/ FFOE/ UFC. Ex-bolsista CAPES. Fortaleza-CE. Brasil. E-mail: kmillamg@yahoo.com.br; Enfermeira. Pós-Doutora em Enfermagem -UNL - Portugal. Professora associada 3 do Programa de Pós-Graduação em Enfermagem do DENF/ FFOE/ UFC. Orientadora da Dissertação. Fortaleza-CE. Brasil. E-mail: zmfca@fortalnet.com.br 


\section{INTRODUÇÃO}

0 cérebro humano há muito tempo vem sendo alvo de estudos, porém muitas perguntas ainda permanecem sem respostas. Ele comanda todas as funções do nosso corpo, como os movimentos, o pensamento e os sentidos. Uma agressão a ele, mesmo que pequena, pode trazer consequências irreversíveis para o organismo. Diante disso, percebemos que alterações como as causadas pela paralisia cerebral (PC) podem levar a grandes limitações para os indivíduos acometidos, conforme veremos adiante.

A PC consiste em um misto de síndromes clínicas e é caracterizada por distúrbios motores e alterações na postura. É uma patologia não progressiva que ocorre durante 0 desenvolvimento fetal ou infantil do cérebro e que pode estar ou não associada a alterações cognitivas. As variações motoras são geralmente acompanhadas por distúrbios na percepção, cognição, comunicação e/ou comportamentos e/ou crises convulsivas. ${ }^{1}$

Por ser um distúrbio que afeta os indivíduos no começo da vida, geralmente ao nascer, e pelo seu caráter incapacitante, provoca forte abalo nas famílias que recebem a notícia de seu diagnóstico. Tal doença causa grandes limitações nas crianças e, consequentemente, na família. ${ }^{2}$ Assim, o nascimento de uma criança com PC faz com que a família se depare com uma situação nova e assustadora, principalmente por ter o potencial de grande visibilidade, aumentando o receio da família quanto à aceitação da criança pela sociedade.

Quando se trata de doenças em crianças, os sentimentos podem ser mais intensos em decorrência de questões culturais de proteção aos filhos e aos laços que se formam desde a gestação. A doença da criança representa um impacto na vida dos pais e familiares. Esta experiência provoca desgaste físico e psicológico, principalmente para o cuidador, que pode ter reações, como o aparecimento do medo, insegurança, depressão, entre outros. ${ }^{3}$

Com efeito, os familiares ou responsáveis por pessoas com qualquer tipo de deficiência também se tornam pessoas com necessidades especiais, uma vez que necessitam de orientações sobre como lidar com o problema, sobre a reorganização da estrutura familiar, as estratégias de enfrentamento e ainda no que concerne ao fato de lidar com os anseios advindos da proximidade com a doença e suas sequelas.

Dentro desse contexto familiar, destacamos aqui a figura da mãe, pois culturalmente a mulher ainda é detentora dos cuidados domésticos e dos filhos, ficando muitas vezes para ela a total responsabilidade pelo cuidado à criança especial. Assim, tendo em vista o compromisso emocional com a criança, é a mãe quem comumente absorve todos os problemas que acometem o filho nesse momento. ${ }^{4}$

A saúde psicológica e física dessa mãe pode estar fortemente influenciada pela gravidade da doença da criança e pela demanda de cuidado. Assim, o difícil processo de cuidar de uma criança com PC, aliado ao crescimento das responsabilidades que essa função promove, pode reduzir seu tempo livre, alterar sua situação profissional e elevar a sobrecarga financeira, bem como levar ao cansaço, isolamento, frustração, sobrecarga e estresse dos cuidadores. Dessa forma, o cuidador da criança com PC, que normalmente é a mãe, altera sua vida em função do maior confor to da criança, passando a não desenvolver efetivamente os seus papéis sociais. ${ }^{5}$

É nesse delicado contexto que os profissionais de enfermagem devem atuar, pois, em razão da sua grande proximidade com os pacientes e familiares, devem estar preparados para oferecer informações com vistas a direcionálos quanto aos cuidados necessários à criança com necessidades especiais. Para isso, o profissional necessita de uma base teórica que Ihe forneça subsídios para orientar e dar apoio à família, dirimindo suas dúvidas e reduzindo suas incertezas quanto ao futuro de uma criança que durante toda a vida dependerá de cuidados especiais de alguém.

A Enfermagem, ao longo dos anos, vem moldando seus conhecimentos a fim de aperfeiçoar a prestação de cuidados aos seus clientes, reduzindo ao máximo os riscos e elevando sua qualidade de vida. 0 conhecimento na Enfermagem pode ser dividido em três períodos: no primeiro, predominaram as técnicas de enfermagem; no segundo, os princípios científicos e a introdução das ciências humanas e, no terceiro, desenvolveram-se as teorias. ${ }^{6}$

Teoria é uma articulação organizada, coerente, sistemática e comunicada em um todo significante da realidade, descoberta ou inventada com vistas a descrever, explicar e predizer situações ou relações. Já a teoria de Enfermagem é definida como a conceituação de alguns aspectos da profissão com a finalidade de descrever, explicar, prever ou prescrever o cuidado. $^{7}$

Surgidas na década de 1950, as teorias de Enfermagem procuraram articular os fenômenos entre si e explicar a realidade, de modo orgânico e coerente. No Brasil, as teorias de Enfermagem desenvolveram-se duas décadas depois, com a publicação do trabalho Contribuição a uma Teoria sobre Enfermagem, da enfermeira Wanda de Aguiar Horta.?

Dentre as diversas teorias no campo da enfermagem, destacamos a Teoria da Incerteza na Doença, da enfermeira norte-americana Merle Helaine Mishel, que foi publicada pela primeira vez em 1981 e coincidiu com o período em que foi testemunha de um câncer de cólon que acometeu seu pai. Nesse momento, observou que os questionamentos dele refletiam diretamente em sua saúde. ${ }^{8}$

Tal teoria trata das incertezas, anseios e dúvidas dos próprios indivíduos doentes, dos cuidadores e dos pais de crianças doentes quanto a um problema de saúde, seja ele agudo ou crônico e que provoca estresse por parte de quem sofre, por não saber o que pode lhe acontecer no futuro. Nesse contexto, a incerteza na doença é um estado cognitivo, no qual o indivíduo é incapaz de atribuir um significado aos 
acontecimentos relacionados com a doença. Nela, uma situação avaliada como incerta mobilizará os indivíduos no uso de seus recursos para se adaptarem à situação. ${ }^{8}$

A incerteza surge no momento em que a pessoa não pode estruturar ou categorizar adequadamente os casos de enfermidade, em razão da falta de significados suficientes. Porém, a incerteza sempre é seguida pelos mecanismos de enfrentamento, que levam à adaptação, resultado desejado ao final do processo. ${ }^{9}$

A Teoria da Incerteza dispõe de três instrumentos para avaliar a incerteza na doença, a saber: A escala para o adulto, usada com adultos hospitalizados; a comunitária, que é usada pelo próprio indivíduo ou por familiares de pessoas cronicamente doentes, mas que não estão hospitalizadas; e a escala na versão de pais/filhos. Nesta, a percepção dos pais sobre a incerteza na doença mede a experiência da incerteza vivenciada pelos pais a respeito da doença de seu filho. ${ }^{8}$

A versão pais/filhos é baseada em quatro pressupostos. 0 primeiro, conhecido como ambiguidade, refere-se à dificuldade no planejamento e na realização dos cuidados à criança. 0 segundo diz respeito à falta de clareza com que se recebem as informações sobre o tratamento da criança. Já o terceiro faz menção à falta da informação a respeito do diagnóstico e da gravidade da doença. Por último, a imprevisibilidade é concernente à falta de habilidade em fazer as previsões diárias ou futuras a respeito da sintomatologia da doença. ${ }^{8}$

A ambiguidade, característica mais geral da incerteza, é caracterizada como a falta de habilidade para se realizar uma atividade previamente planejada de cuidados à criança, 0 que impede os pais de traçarem planos, tanto em curto quanto em longo prazo. Sendo assim, quando os estímulos são julgados como ambíguos, surge a incerteza. A diversidade de tratamentos oferecidos à criança é um exemplo de como a ambiguidade pode atuar, pois os pais, muitas vezes, não possuem a habilidade em distinguir entre um e outro tratamento oferecido. ${ }^{8,10}$

No estudo ora apresentado, será tratado somente 0 aspecto da ambiguidade. Todavia, uma versão completa trata de todos os pressupostos presentes na versão direcionada aos pais em relação ao cuidado com os filhos.

0 aprofundamento desse estudo é viável a fim de que seja feita a correlação entre as teorias de Enfermagem e a questão da convivência com a paralisia cerebral, uma vez que poderá ser uma contribuição para a melhoria na qualidade de vida dos familiares, refletindo diretamente nos portadores de paralisia cerebral. Permitirá também aos profissionais de enfermagem outra perspectiva, acerca do cuidar não só na PC como também com todas as outras doenças crônicas e/ou incapacitantes. Permitirá, ainda, o exercício da visão holística dos profissionais, situando também o cuidador como objeto de atenção dos profissionais, e não apenas a criança e sua doença.

Assim, na tentativa de ampliar a base teórica dos enfermeiros, resolvemos desenvolver este estudo, porquanto é evidente a carência de assuntos que vinculam a qualidade de vida com a incerteza no cuidado a crianças com necessidades especiais, bem como às teorias de Enfermagem. Com efeito, 0 cuidado a essa crianças é procedido, muitas vezes, de forma empírica e sem levar em consideração o lado social, cultura e emocional da família envolvida.

Com base no exposto, este ensaio tem como objetivo analisar, na percepção das mães, a incer teza na doença de seus filhos com paralisia cerebral com base no pressuposto da ambiguidade da Teoria da Incerteza na Doença, de Mishel.

\section{METODOLOGIA}

Optamos por realizar uma pesquisa descritiva na perspectiva da investigação qualitativa, uma vez que nosso intuito foi conhecer, por meio dos depoimentos das mães, suas concepções sobre 0 tratamento de seus filhos. A pesquisa qualitativa consiste na investigação de fenômenos, de forma profunda e holística, através da coleta de materiais narrativos ricos, usando um delineamento de pesquisa flexível. Já o tipo descritivo de pesquisa refere-se a estudos que possuem como principal objetivo o retrato preciso das características de indivíduos, situações ou grupos e da frequência com que ocorrem determinados fenômenos. ${ }^{11}$

0 estudo foi realizado em duas instituiç̧ões da rede publica de Fortaleza, a saber: Núcleo de Tratamento e Estimulação Precoce - NUTEP e o Hospital Infantil Albert Sabin - HIAS. A escolha recaiu nessas duas instituições visto que são referências terciárias no atendimento de reabilitação infantil no Município de Fortaleza e no Estado do Ceará. A decisão por fazer a pesquisa em dois ambientes decorreu do fato do reduzido número de crianças sendo atendidas na faixa etária proposta no estudo. Dessa forma, por medidas cautelares, optamos por dois serviços, a fim de alcançar um número satisfatório de entrevistadas.

Participaram da pesquisa 12 mães de crianças com 0 diagnóstico de paralisia cerebral. Tal escolha foi feita, uma vez que, de modo geral, as mães se envolvem mais no processo de tratamento, indo com mais frequência ao hospital e interagindo com a equipe de profissionais que tratam da criança, ${ }^{12}$ fato constatado por ocasião das visitas aos serviços, onde somente as mães acompanhavam seus filhos.

A participação de cada mãe foi definida a partir dos critérios de inclusão: ter recebido o diagnóstico médico de PC há um ano ou menos, pois acreditamos que as mães que receberam a notícia há mais tempo já tenham tido tempo para uma melhor adaptação do que as que tenham recebido nesse período; ser a cuidadora principal; fazer acompanhamento contínuo nas instituições cenários do estudo.

Foi considerado critério de exclusão: mães que ainda não haviam recebido o diagnóstico médico de $P C$ até 0 momento da seleção das envolvidas na amostra ou crianças acompanhadas por outros parentes que não a mãe. 
A coleta dos dados foi realizada entre os meses de julho e agosto de 2009, tendo sido utilizados dois instrumentos: um formulário com as variáveis sócio-econômico-sanitáriodemográficas das mães e um roteiro de entrevista semiestruturada com dados relativos à incerteza na doença.

As perguntas do roteiro de entrevista foram baseadas na Escala da Incerteza versão pais/filhos da Teoria da Incerteza na Doença, de Mishe ${ }^{10}$, que está dividida nos quatro pressupostos anteriormente citados. Para a consecução desse artigo, selecionamos o pressuposto da ambiguidade, que se refere à indeterminação sobre o planejamento diário de cuidado à criança. ${ }^{8}$

A entrevista foi composta primeiramente por dados de identificação (idade, procedência, estado civil, escolaridade, profissão, etc.), lembrando que o nome ou endereço da entrevistada não fizeram parte da entrevista. Em seguida, foram investigados os dados referentes ao pressuposto da ambiguidade, da Teoria da Incerteza na Doença.

Antes de iniciar a coleta oficial dos dados, realizamos 0 pré-teste dos instrumentos com três mães, as quais não fizeram parte da amostra. Isso permitiu a inclusão de questões importantes e os ajustes necessários, evitando lacunas.

0 pré-teste é um ensaio para determinar se o instrumento foi formulado com clareza, com imparcialidade, e se é útil para a geração das informações desejadas. Ainda, é a administração, em forma de tentativa, de um instrumento recém-elaborado para identificar falhas ou avaliar as exigências de tempo. ${ }^{11}$

As entrevistas foram realizadas no momento em que a criança estava sendo atendida por algum dos profissionais, momento em que a mãe aguardava em sala de espera. Foi escolhido um local reservado, uma vez que poderia ficar intimidada com as outras pessoas presentes, dificultando o bom andamento da entrevista.

Não houve delimitação de tempo para a realização das entrevistas, porém estas tiveram duração média de 20 minutos. Aconteceram nas instituições onde as mães tratam seus filhos, sendo cada uma no dia e horário preestabelecidos entre nós e a mãe, em local reservado, de acordo com a anuência do serviço. Foi utilizado um gravador, com a permissão da participante da pesquisa, com o objetivo de garantir fidedignidade da reprodução do pensamento e permitir maior atenção às expressões não verbais dos entrevistados, visto que estaríamos livres de anotaç̃̃es durante o diálogo.

Os dados foram organizados por meio da Análise de Conteúdo, a qual consiste em um conjunto de técnicas de análise das comunicações, visando a obter, por procedimentos sistemáticos e objetivos, a descrição do conteúdo das mensagens (quantitativos ou não) que permitam a inferência de conhecimentos relativos às condições de produção/recepção (variáveis inferidas) destas mensagens. ${ }^{13}$

São três as fases básicas para trabalhar com a análise de conteúdo: Pré-análise, na qual foi feita a escuta, transcrição e leitura das entrevistas; Exploração do material, com a separação das frases por categorias temáticas; e Tratamento dos resultados, inferências e interpretação, em que foi feita a discussão por categorias. ${ }^{13}$

0 projeto deste artigo foi enviado aos Comitês de Ética em Pesquisa da Maternidade-escola Assis Chateaubriand e Hospital Infantil Albert Sabin, em concordância com as determinações da resolução 196/96, que define as diretrizes que regem a pesquisa com seres humanos, ${ }^{14}$ tendo sido aprovado sob os números 058/09, de 02/09/2009 e 012/09, de 16/ 04/2009, respectivamente.

Antes das entrevistas, as mães foram devidamente esclarecidas quanto à finalidade do estudo e quanto à importância de sua participação. Após a explicação e aceitação por parte destas, assinaram o Termo de Consentimento Livre e Esclarecido.

0 anonimato das envolvidas esteve assegurado, uma vez que neste relatório os nomes das mães são fictícios, tendo sido inspirados em grandes personagens da literatura brasileira.

Os dados obtidos foram utilizados apenas para a realização deste estudo, tendo a participante da pesquisa a liberdade de retirar seu consentimento a qualquer momento, sem que isto acarretasse problemas no atendimento da criança na unidade.

\section{RESULTADOS E DISCUSSÃO}

Aamostra estudada foi formada por 12 mães de crianças com o diagnóstico de paralisia cerebral e que se encontravam nos serviços por ocasião da consulta do filho. A maior parte delas, sete, reside na capital. Onze destas são donas de casa, abrangendo as faixas etárias entre 16 e 39 anos. Apenas quatro concluíram o ensino médio, sendo que cinco não chegaram a concluir o ensino fundamental II. Oito são católicas e quatro evangélicas. A renda mensal da maioria das mães esteve entre 1 e 2 salários mínimos da época, isto é, entre $R \$ 465,00$ e $R \$$ 930,00. A composição familiar variou entre três e treze pessoas. Sete mães tinham somente um filho, com idade variando entre dez meses e três anos, sendo que o diagnóstico de todas elas foi dado há um ano ou menos, variando entre três meses e um ano de conhecimento da situação de PC dos filhos.

Diante da descrição acima, percebe-se que, em sua quase totalidade, essas mães fazem parte de uma população vulnerável, de baixa renda e escolaridade, relações conjugais instáveis e que dependem financeiramente de alguém por não terem emprego remunerado. As expectativas das famílias das crianças com paralisia cerebral estão diretamente ligadas ao contexto social e à sua condição econômica. Além disso, sua visão de mundo é referenciada pelo contexto em que vivem. ${ }^{15}$

Praticamente todas as crianças participam das atividades de fisioterapia, terapia ocupacional e fonoaudiologia, bem como avaliações periódicas com a equipe multidisciplinar para averiguar seu estado geral e sua evolução.

A investigação feita às mães participantes da amostra foi referente ao pressuposto da ambiguidade, que avalia questões como as implicações futuras do diagnóstico 
para mãe-filho, possíveis alterações nos planos traçados para o futuro da família, o apoio de outras pessoas no cuidado à criança e a possível melhoria com o tratamento e as medicações.

Quando investigadas sobre as implicações que o diagnóstico de $\mathrm{PC}$ pode trazer para a vida do binômio mãefilho, uma delas fez a seguinte colocação:

Ah! Eu penso demais nisso. Já pensou se ele não andar nunca? Se não falar... Quer dizer, a gente que émãe sonha o melhor para o seu filho. Aíde repente nasce um neném doentinho, fraquinho, que precisa de todo tipo de cuidado... Dá um medo danado! Vai mudar tudo na nossa vida! Eu já vivo só pra ele, mas eu sei que vai ser difícil pra todo mundo. (Carolina)

No depoimento, percebemos que a família é geralmente confrontada com novas exigências, alterações nas suas rotinas, mudanças constantes e readaptações diversas, propiciando a que a doença propague seus efeitos nos níveis financeiro, ocupacional, pessoal e social. ${ }^{16}$

As incertezas das mães ante a nova condição em que se encontram foram identificadas como uma das situações que contribuem para seu estresse, uma vez que seus conceitos a respeito do futuro, tanto delas quanto dos filhos, ainda é incerto, impedindo-as, muitas vezes, de elaborar metas. ${ }^{10}$

Assim, as mães participantes, responsáveis por praticamente todos os cuidados à criança, não somente tiveram suas rotinas modificadas com o nascimento de uma criança com necessidades especiais, mas também uma parte de seu futuro se tornou incerta em decorrência das incertezas quanto ao cuidado diário a uma criança que necessita de atenção especial em tempo integral.

Para famílias de crianças com PC, principalmente pelo caráter crônico da doença, as alterações no estilo de vida podem ser enormes. Essas famílias têm alteração em sua dinâmica, rompendo esquemas educativos e alterando relações na vida afetiva. ${ }^{17}$ Nesse sentido, planos anteriormente traçados pela família necessitam mudar de configuração em virtude do nascimento da criança doente. A fim de investigar essa temática, perguntamos às mães se houve alguma mudança nos planos traçados para o futuro da família com o nascimento da criança com necessidades especiais. Uma das respostas está descrita a seguir.

Meus planos eram de trabalhar. Eu sempre gostei! Aíagora com ele assim, eu não posso nem trabalhar. Eeu também tenho medo de deixar ele com alguém. Aía minha vida é só pra ele mesmo. (Cecilia)

Quando ocorre uma situação como essa, do nascimento de uma criança com necessidades especiais, normalmente quem sacrifica sua rotina é a mãe. Muitas delas deixam seus empregos, interrompem parte de sua vida social para dedicar-se, exclusivamente, ao cuidado de seu filho. A abdicação de sua vida profissional altera a sua rotina e influencia a família como um todo, pois, ao sair de seu emprego, além de reduzir a renda familiar, passa a depender financeiramente de outros. ${ }^{18}$

0 processo de cuidar de uma criança com PC exige do cuidador grandes responsabilidades, uma vez que a criança necessita, em sua maioria, de cuidados de forma integral em decorrência das limitações impostas pela doença. 0 cuidador, porém, que, no caso desta pesquisa, está representado em sua totalidade pelas mães, em virtude da responsabilidade quase integral de cuidados à criança, pode se encontrar sobrecarregado em decorrência da prestação de cuidados à criança e, com isso, ter reduzida a sua qualidade de vida. Nesse contexto, perguntamos às mães se recebiam apoio de alguém nas tarefas do lar. Uma delas fez a colocação a seguir:

\section{Não. É mais eu mesma. Até pra cá eu venho só com ela. Trago bolsa, trago guarda-chuva... até o pessoal aqui fica tudo olhando e perguntando como é que eu aguento. Mas é porque é assim mesmo. Aíláe é tudo eu. É eu que vou pra médico. Se tem que levar é eu que levo.( Amália)}

Pela fala dessa mãe, percebemos que a prestação de cuidados à criança é uma missão de total responsabilidade da mãe. Portanto, o que acontece, na maioria das vezes, é o cuidado em tempo integral à criança. Raramente a responsabilidade da prestação de cuidados é partilhada por mais de uma pessoa, além de, também, raramente corresponder a uma situação de escolha assumida. ${ }^{15}$

Por último, perguntamos às mães se houve alguma melhoria da criança após o início do tratamento e da administração das medicações. Uma delas deu a seguinte resposta:

[...] quando eu dou o remédio, ele já melhora. E em relação ao tratamento, ele melhorou também, porque ele num saía nem do canto. Botava ele na cama e era todo tempo parado. Isso com 7 meses! Aí depois que ele veio pra cá, num instante! E ele já está começando a falar [...] que antes ele não falava! (Carolina)

Percebemos que esta mãe se encontra em uma situação de maior segurança em relação aos resultados positivos apresentados pelo filho com o suporte do tratamento oferecido pelo serviço, bem como da medicação dada. Para ela, o sacrifício despendido em deslocamento até o lugar de tratamento e todas as outras exigências impostas podem estar sendo compensados mediante 0 desenvolvimento, ainda que pequeno e demorado, de seu 
filho. Com isso, além de renovadas as esperanças quanto à saúde da criança, essas mães têm suas incertezas reduzidas em virtude dos resultados.

\section{CONSIDERAÇÕES FINAIS}

A realização deste estudo permitiu a descrição das múltiplas facetas do cuidar no contexto domiciliar. 0 trabalho ora apresentado avalia, por meio de dados apreendidos, a realidade vivenciada pelas mães envolvidas no cuidar destas crianças e fornece a exploração do pressuposto da ambiguidade da Teoria da Incerteza na Doença.

Os achados do estudo mostram o receio das mães quanto ao futuro da criança, podendo interferir nas decisões de toda a rede familiar; mostra também que existe uma colaboração parcial ou inexistente de outras pessoas no cuidado às crianças, trazendo sequelas físicas e psicológicas para essas mães cuidadoras; revela ainda a repercussão positiva da melhoria do quadro de saúde das crianças com o tratamento, o que reflete na redução das incertezas das mães.

Expressas as considerações acima, constatamos que essas mães possuem ainda muitos sentimentos de incer teza quanto à patologia da criança, principalmente quanto ao futuro. Tais incertezas podem vir a ser reduzidas mediante apoio dos profissionais, que, tendo melhor manejo com as mães, tirando suas dúvidas, dando explicações sobre a patologia e todo 0 processo de tratamento, concedendo um feedback quanto à evolução da criança, podem reduzir as dúvidas que possuam em virtude da doença.

Por fim, a PCé uma alteração que não só causa distúrbios neuromotores no indivíduo, mas também leva a profundas alterações psicológicas e sociais nas pessoas que com ele convivem, principalmente as mães, foco principal de nosso estudo.

\section{REFERÊNCIAS}

1.Rosenbaum P, Paneth N, Leviton A, Goldstein M, Bax M, Damiano D, et al. A report: the definition and classification of cerebral palsy April 2006. De. Med Child Neurol. 2007; 109 (1): 8-14.

2.Rocha DLB, Zagonel IPS. Modelo de cuidado transicional à mãe da criança com cardiopatia congênita. Acta Paul Enferm. 2009 jun; 22 (3): 243-49.

3. Gondim KM, Pinheiro PNC, Carvalho ZMF. Participação das mães no tratamento dos filhos com paralisia cerebral. Rev Rene. 2009 out/dez; 10(4): 136-44.

4.Camargos ACR, Lacerda TTB, Viana SO, Pinto LRA, Fonseca MLS. Avaliação da sobrecarga do cuidador de crianças com paralisia cerebral através da escala Burden Interview. Rev Bras Saude Matern Infant. 2007 jan/mar; 9 (1): 31-7.
5.Almeida MCP, Rocha JSY. 0 saber de enfermagem e sua dimensão prática. São Paulo: Cortez; 1986.

6.Meleis Al. TnNursing: development \& progress. $3^{\mathrm{a} e d .}$ Philadelphia: Lippincott; 1997.

7.Bailey Jr DE, Stewart JL. Merle Mishel: La incertidumbre frente a la enfermedad. In: Tomey AM, Alligood MR. Modelos y teorías de enfermería. Barcelona: Elservier Science; 2003. p. 561-84.

8.Mishel MH. The measurement of uncertainty in illness. Nurs Res. 1981; 30 (5): 258-63.

9. Torres A, Sanhueza 0. Modelo estructural de enfermería de calidad de vida e incertidumbre frente a la enfermedad. Cienc Enferm. 2006 jun; 12 (1): 9-17.

10.Mishel MH. Parents perception of uncertainty concerning their hospitalized children. Nurs Res. 1983 Nov/Dec; 32 (6): 324-30.

11.PolitDF,BeckCT, Hungler BP. Fundamentos de pesquisa em enfermagem: métodos, avaliação e utilização. $5^{\text {a }}$ ed. Porto Alegre: Artmed; 2004.

12.Prudente COM, Barbosa MA, Porto CC. Relação entre a qualidade de vida de mães de crianças com paralisia cerebral e a função motora dos filhos após dez meses de reabilitação. Rev Latino-am Enfermagem. 2010 abr; 18 (2): 149-55.

\section{Bardin L. Análise de conteúdo. Lisboa: Ed 70; 2009.}

14.Ministério da Saúde (BR). Conselho Nacional de Saúde. Comissão Nacional de Ética em Pesquisa. Resolução n.196, de 10 de outubro de 1996: aprova as diretrizes e normas regulamentadoras de pesquisa envolvendo seres humanos. Brasília(DF); 1996.

15.Ferreira HBG. Aspectos familiares envolvidos no desenvolvimento de crianças com Paralisia Cerebral [dissertação]. Ribeirão Preto: Departamento de Medicina Social, Universidade de São Paulo; 2007.

16.Santos SV. A família da criança com doença crônica: abordagem de algumas características. Aná Psicológica. 1998 mar; 1 (14): 65-75.

17.0rtiz MCA. Experiências de pais de crianças e adolescentes após o término do tratamento de câncer: subsídios para o cuidado de enfermagem [dissertação]. Ribeirão Preto: Escola de Enfermagem, Universidade de São Paulo; 2006.

\section{NOTA}

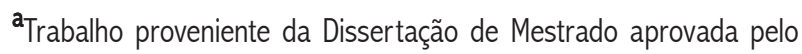
Programa de Pós-Graduação em Enfermagem da Faculdade de Farmácia, Odontologia e Enfermagem da Universidade Federal do Ceará PPGENF/ DENF/FFOE/UFC. Contou com o apoio financeiro em forma de Bolsa da Coordenação de Aperfeiçoamento de Pessoal de Nível Superior - CAPES. 